\title{
Untangling the Effect of Fatty Acid Addition at Species Level Revealed Different Transcriptional Responses of the Biogas Microbial Community Members
}

Treu, Laura; Campanaro, Stefano; Kougias, Panagiotis; Zhu, Xinyu; Angelidaki, Irini

Published in:

Environmental Science and Technology

Link to article, DOI:

10.1021/acs.est.6b00296

Publication date:

2016

Document Version

Peer reviewed version

Link back to DTU Orbit

Citation (APA):

Treu, L., Campanaro, S., Kougias, P., Zhu, X., \& Angelidaki, I. (2016). Untangling the Effect of Fatty Acid Addition at Species Level Revealed Different Transcriptional Responses of the Biogas Microbial Community Members. Environmental Science and Technology, 50(11), 6079-6090. https://doi.org/10.1021/acs.est.6b00296

\section{General rights}

Copyright and moral rights for the publications made accessible in the public portal are retained by the authors and/or other copyright owners and it is a condition of accessing publications that users recognise and abide by the legal requirements associated with these rights.

- Users may download and print one copy of any publication from the public portal for the purpose of private study or research.

- You may not further distribute the material or use it for any profit-making activity or commercial gain

- You may freely distribute the URL identifying the publication in the public portal 
Untangling the effect of fatty acids addition at species level revealed different transcriptional responses of the biogas microbial community members

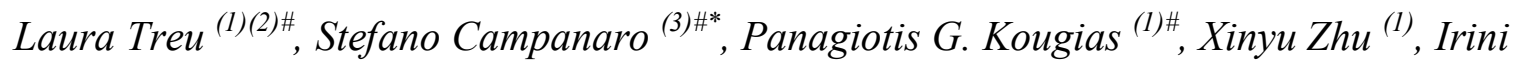
Angelidaki $^{(1)}$

(1) Department of Environmental Engineering, Technical University of Denmark, Kgs. Lyngby, Denmark

(2) Department of Agronomy Food Natural Resources Animals and Environment (DAFNAE), University of Padova, Viale dell'Università 16, Legnaro, PD 35020, Italy

(3) Department of Biology, University of Padova, Via U. Bassi 58/b, 35121, Padova Italy

KEYWORDS methane, anaerobic digestion, methanogens, metatranscriptomic, RNA sequencing, long chain fatty acids, oleate. 
ABSTRACT In the present study, RNA-sequencing was used to elucidate the change of the anaerobic digestion metatranscriptome after long chain fatty acids (oleate) exposure. In order to explore the general transcriptional behavior of the microbiome, the analysis was firstly performed on the shotgun reads without considering a reference metagenome. As a second step, the RNA reads were aligned on the genes encoded by the microbial community, revealing the expression of more than 51,000 different transcripts. The present study is the first research which was able to dissect the transcriptional behavior at a single species level by considering the 106 microbial genomes previously identified. The exploration of the metabolic pathways confirmed the importance of Syntrophomonas species in fatty acids degradation, and also highlighted the presence of protective mechanisms towards the long chain fatty acid effects in bacteria belonging to Clostridiales, Rykenellaceae and in species of the genera Halothermothrix and Anaerobaculum.

Additionally, an interesting transcriptional activation of the chemotaxis genes was evidenced in seven species belonging to Clostridia, Halothermothrix and Tepidanaerobacter. Surprisingly, methanogens revealed a very versatile behavior different from each other, even among similar species of the Methanoculleus genus, while a strong increase of the expression level in Methanosarcina sp. was evidenced after oleate addition.

\section{INTRODUCTION}

Biogas production is a sustainable solution for energy generation and waste resource recovery, and therefore, attracted increasing attention from the society. Biogas is generated through the Anaerobic Digestion (AD) of organic matter, which is accomplished by an intricate set of microbial species. In previous studies, this complex microbiome was studied mainly using traditional microbiological techniques or targeting the $16 \mathrm{~S}$ rRNA gene; thus, emphasizing on the phylogenetic characterization of the microbiome ${ }^{1-3}$. Only recently, metagenomic studies were performed focusing on the genome and metagenome characteristics in order to improve the phylogenetic analysis and additionally to determine the functional roles of the microbial species

in the $\mathrm{AD}$ system ${ }^{4,5}$. In these studies, the assembly and binning processes of the high throughput sequencing reads can benefit further metatranscriptomic research. First of all, it is known that the assembly of the shotgun reads leads to a more solid analysis of the high throughput sequencing reads, which are otherwise 
error-prone ${ }^{6}$. This procedure improves the gene finding process and, consequently, allows the alignment of the RNA-seq reads on genes predicted from the assembly, providing a more reliable calculation of their expression level. Secondly, the binning process is of a great importance because it untangles the microbial genomes from the metagenomic assembly ${ }^{7}$. One of the main limitations of the short-reads is the minimal signal usable for homology searches. This restriction hampers direct annotation against reference databases, but it can be overcome by the binning process that allows the assignment of the reads obtained from the RNA-seq at the species-level. This last step uncovers the transcriptional behavior of the single species in the microbial community and reveals the transcriptionally inactive species ${ }^{8}$. As it can be easily understood from the above, the gene prediction and annotation processes allow the identification of the metabolic pathways that a species can potentially utilize; nevertheless, only a transcriptional study can really determine which genes undergo transcriptional activation in a specific environmental context. Therefore, a metatranscriptomic approach will significantly contribute to elucidate the functional role of the biogas microbial community.

Despite the advantages of the metatranscriptomics, very few gene expression studies were previously performed on the $\mathrm{AD}$ microbiome using next-generation sequencing ${ }^{9,10}$. All of these analyses were mainly focused on a general definition of the active part of the biogas community without exploring the transcriptional changes determined by an environmental stimulus. Additionally, the analyses performed in previous studies with the Genome Sequencer FLX instrument were strongly limited by the "low" throughput of this sequencing platform, which range around 0.5-1 million reads ${ }^{10}$. Out of the total number of sequences, mRNA represents a minor fraction of the total RNA $(\sim 5 \%)$ resulting in the identification of a few thousands of reads assigned to the mRNA transcripts. It has also to be considered that rRNA subtraction is particularly difficult in the metagenomics samples, due to the high diversity of the species present, and gives at best a fourfold increase in the recovery of non-rRNA reads ${ }^{11}$. For all these reasons, it is still unclear to what extent high-throughput sequencing methods provide a representation of the expressed functional genes in microbial community transcriptomes. Despite these limitations, the metatranscriptomic analysis of the anaerobic digester community revealed transcripts encoding enzymes involved in substrate hydrolysis, acidogenesis and acetate formation, and evidenced a high transcriptional activity of archaeal population ${ }^{10}$. These results were confirmed by a more recent study performed using a high sequencing depth, that also revealed a high expression level for the genes involved in hydrogenotrophic methanogenesis in the single reactor analyzed ${ }^{9}$. 
However, in the majority of the previously cited metatrascriptomic studies, a large proportion of reads could not be assigned to known species, highlighting the need for a reference genome/metagenome.

In the present research, a comprehensive metatranscriptomic analysis was performed in triplicate biogas reactors that underwent radical shifts of the influent feedstock induced by the addition of Long Chain Fatty Acids (LCFA). The alignment of the RNA-seq reads on genes and corresponding genomes was supported by a previous metagenomics study performed by the authors and focused on metagenomics assembly and binning of the microbial community in laboratory scale biogas reactors ${ }^{5}$. This previous analysis was performed before and after LCFA addition and led to the identification of the 106 GBs analyzed in the present metatranscriptomic project. The addition of the unsaturated LCFA in the feedstock as a mean to alter the community dynamicity was specifically selected due to the significant importance of such compounds in the AD process. LCFA are primarily degraded via $\beta$-oxidation generating acetate and hydrogen, which are further converted to methane and carbon dioxide. This process is concertedly performed by fatty acids oxidizing bacteria and methanogens ${ }^{12}$. The bacterial counterpart is commonly represented by protonreducing acetogenic bacteria requiring the syntrophic interaction with $\mathrm{H}_{2}$-utilizing methanogens and acetoclastic methanogens ${ }^{13}$. Thus far all the bacterial species that are able to grow on fatty acids with 4-18 carbon atoms belong to Syntrophomonadaceae, Syntrophaceae and Clostridiaceae families ${ }^{14-17}$. The archaeal species that are more frequently involved in this syntrophic cooperation are the hydrogenotrophic methanogens belonging to Methanobacterium genus, but also some acetoclastic archaea (i.e.

Methanosarcina) have been identified ${ }^{18-20}$. This syntrophic interaction has been extensively studied, and also some analyses investigated the transcriptional changes involved in lipid utilization in Syntrophomonas species ${ }^{21-23}$. Furthermore, modifications of the microbiome determined by inhibitory levels of LCFA have been previously reported ${ }^{24-26}$. Despite this, no metatranscriptomic studies were performed to elucidate the activity of the AD microbial community as a result of LCFA addition. Moreover, it is still unclear whether the main determinant of this process is a shift towards better adapted LCFA-degraders or the phenotypic adaptation of the existing microorganisms towards high LCFA concentrations (physiological acclimatization). 
To the best of our knowledge this is the first research elucidating the activity of the AD microbiome in adaptation to LCFA focusing on how the microbial species react to the LCFA addition by exploring the changes from a transcriptional point of view.

\section{MATERIALS AND METHODS}

\section{Biogas reactors' configuration and sample collection.}

The experiment was carried out in triplicate continuous stirred tank reactors (CSTR), denoted as CSTR01, CSTR02 and CSTR03. The reactors had total volume of $2 \mathrm{~L}$ and working volume of $1.5 \mathrm{~L}$. All reactors were continuously stirred using magnetic stirrers and were equipped with thermal jackets in order to maintain the operating temperature steady at $54 \pm 1{ }^{\circ} \mathrm{C}$. In the beginning all reactors were inoculated with thermophilic inoculum obtained from Snertinge biogas plant, Denmark. The experiment was divided in two periods; (1) during the first period all the reactors were fed exclusively with cattle manure (CM), and (2) during the second period the influent feedstock of each reactor was radically changed by adding sodium oleate (NaOleate, $12 \mathrm{~g} / \mathrm{L}$-feed) to the CM. The influent feedstock was automatically provided twice per day using peristaltic pumps. Throughout the experiment the hydraulic retention time (HRT) of all reactors was kept constant at 15 days. Samples for metatranscriptomic analyses ( $15 \mathrm{ml}$ each) were collected from all reactors during the steady state condition of each period (i.e. period with stable biogas production with a daily variation lower than $10 \%$ for at least 5 days). The three samples (biological replicates) obtained from the first period were indicated as CSTR01a, CSTR02a and CSTR03a, while the three samples obtained from the second period were indicated as CSTR01b, CSTR02b and CSTR03b.

\section{RNA extraction and sequencing.}

Barley residues present in the manure were removed using a $100 \mu$ m nylon cell strainer filter as previously described ${ }^{27}$. The filtered sample was centrifuged at 5,000 rpm for $10 \mathrm{~min}$ and the supernatant was discarded leaving $\sim 2 \mathrm{~g}$ of material. To avoid RNA degradation, $3.5 \mathrm{ml}$ of Phenol: Chloroform ( $\mathrm{pH}$ 6.7/8.0) premixed with isoamyl alcohol (25:24:1) (Amresco, Incorporated) was added to the pellet after centrifugation and the samples were immediately further processed for RNA extraction. Only few minutes elapsed between samples collection and the addition of phenol solution. Total RNA was extracted from these $2 \mathrm{~g}$ of material 
using the RNA PowerSoil ${ }^{\circledR}$ Kit (MO BIO laboratories, Carlsbad, CA). The quality and the quantity of the extracted RNA were determined both using NanoDrop (ThermoFisher Scientific, Waltham, MA) and Qubit fluorometer (Life Technologies, Carlsbad, CA). RNA integrity was determined using Agilent Bioanalyzer, (Supporting Information, Figure S3). The quality of the samples fulfills the requirements of library preparation and RNA-sequencing of the Ramaciotti Centre for Genomics. A visual representation of the RNA-seq reads on some selected regions of the metagenome assembly assigned to the GB Eu01 is reported in (Supporting Information, Figure S2).

RNA libraries were prepared from individual samples using the TruSeq RNA Library Preparation Kit (Illumina, San Diego, CA). All the samples were paired-end sequenced ( 2 x 150 bp) using MiSeq system (Illumina, San Diego, CA) in two different runs and considered as two technical replicates. Due to the very high coefficient of determination calculated from the comparison of the two technical replicates (Supporting Information, Figure S4), the reads obtained from the two sequencing runs were considered together in the subsequent analyses. By considering the two technical replicates together, in the six samples were obtained 5,723,021-5,908,785-6,286,045-6,871,442-6,696,876-5,494,112 sequences. Reads in FASTQ format were quality-filtered and the adaptors were removed using Trimmomatic software ${ }^{28}$.

\section{Metatranscriptomic analysis with MG-RAST.}

All the reads obtained after filtering were uploaded to the MG-RAST metagenomics analysis server ${ }^{29}$ and are available for download. Experiments are associated to the following accession ID: CSTR01a (4579369.3), CSTR02a (4579370.3), CSTR03a (4579371.3), CSTR01b (4579372.3), CSTR02b (4579373.3), CSTR03b (4579374.3). Reads were analyzed using the following parameters: Max. e-Value Cutoff 1e-5, Min. \% Identity Cutoff 60\%, Min. Alignment Length Cutoff 15. Data were analyzed using a) "subsystem2nd level" analysis, b) "KO-3rd level" analysis and c) "KEGG-orthology functions". Data were visualized as a heatmap where each square represents the abundance level of a single category in a single sample. Numerical values were downloaded and included in the Dataset S2 and average values for the six samples were used to generate figures 1 and 2.

\section{Reads alignment, gene expression calculation and statistical analysis.}


Analysis on the single genes was performed considering the global metagenome assembly (v1) previously determined ${ }^{5}$ and available in the biogas microbiome website (http://www.biogasmicrobiome.com/) and at DDBJ/EMBL/GenBank under the accession LFRM00000000. Filtered reads were aligned on reference metagenome assembly using bowtie $2^{30}$ and the number of reads mapped per each gene was determined from the "sam" file using HTSeq ${ }^{31}$ with the options "-count" and "intersection-non empty". The statistical analysis was performed using edgeR software ${ }^{32}$ and the differentially expressed genes were filtered considering the $\mathrm{p}$-value (pVal.Tgw $<0.05)$ and the coverage ratio $(>2$ fold).

In order to determine the contribution of the genome abundance variation to the changes in gene expression level, the same reads alignment and filtering procedure was applied using the shotgun DNA reads obtained for the same six samples ${ }^{5}$. The variation of the "DNA coverage" between the two time periods (before and after LCFA addition) was determined by changes in abundance of the species in the microbial community. The contribution due to variations of the genome abundance was removed from the gene expression changes; this was performed by comparing the results obtained from edgeR software determined for DNA reads and RNA reads.

Scaffold coverage was used to calculate the species abundance. It was previously determined with the genomecov software of the bedtools package ${ }^{33}$ as described by ${ }^{5}$ using as input the DNA-seq reads aligned to the assembly. Coverage was normalized considering the number of aligned reads and using the sample with the lower number as a reference. Starting from the scaffold coverage, the average coverage of each genome was determined using the "calcolo_genome_coverage.pl” perl script available in http://www.biogasmicrobiome.com. The "absolute expression level", which is defined as the total number of RNA reads assigned to each microorganism was calculated with the same procedure.

KEGG pathway maps in Supporting Information (Figure S1) were obtained with the "KEGG Mapper Search\&Color Pathway" tool ${ }^{34}$. The absolute expression level of genes was represented as a heat map (Figure S1a) and the differentially expressed genes were highlighted in color (Figure S1b, c).

To identify the COG and KEGG functional classes statistically enriched of differentially expressed genes the procedure described in ${ }^{35}$ was followed. 10,000 random samplings of $\mathrm{n}$ genes (where $\mathrm{n}$ is the number of genes for each COG or KEGG class) were performed on the entire dataset of genes expressed (Dataset S3) 
using a perl script implementing the "rand()" function. For each class we calculated the fraction of random samples in which the number of genes differentially expressed was equal to or higher than DE (where DE is the number of genes differentially expressed in the group of $n$ genes). If this fraction was lower than the significance level $\alpha(0.05)$ the enrichment of genes differentially expressed in the $\mathrm{n}$ genes was considered significant.

\section{RESULTS AND DISCUSSION}

Shotgun reads obtained for each sample ranged between 5.5 and 6.9 million (2.5-3.45 million paired-end reads) and were analyzed using two different procedures. The first one was based on a global functional analysis performed on the shotgun reads uploaded to the MG-RAST database and analyzed using "subsystem" and "KEGG". This process is not based on prior knowledge of the metagenomic assembly ${ }^{5}$ and for this reason it is totally independent from the genes previously identified. This approach aimed to obtain a general overview of the transcriptional modification in response to LCFA addition. On the contrary, the second approach described below was based on the alignment of shotgun reads to the metagenomic assembly. This procedure focused the analysis only on the genes previously identified, and has the advantage of "anchoring" the reads to a high quality gene prediction. Furthermore, it can also benefit from the assignment of the reads to the 106 microbial genomes previously identified ${ }^{5}$. These genomes derived from the binning process of the metagenomics assembly and are referred as Genome Bins (GBs). As reported below, this approach is more appropriate to explore the effect of LCFA addition on the different species of the microbial community.

\section{Functional analysis of the reads on MG-RAST database.}

Shotgun sequences (not rRNA subtracted) were uploaded to MG-RAST and analyzed. The overview of the microbial activity relied on a three stage analysis approach, in which the extracted information and interpretation of the data progressed from more generic to more detailed level. More specifically, the transcriptional profile of the biogas microbial community was monitored by a) "subsystem- $2^{\text {nd }}$ level" analysis which provided a general description highlighting the most active genes and the prevalent functions 
(Figure 1), b) "KO-3 ${ }^{\text {rd }}$ level” analysis providing an overview of the KEGG pathways and additionally complemented the previous analysis (Figure 2), and c) "KEGG-orthology functions" analysis revealing genes increasing their expression after the LCFA addition to the reactor. Numerical data for "KEGGorthology" and "subsystem" are reported in Dataset S2.

The "subsystem- $2^{\text {nd }}$ level" analysis" showed that the transcriptional activity was dominated by the "onecarbon metabolism", which includes the genes of methanogenesis pathway (Methane metabolism, ko00680) (Figure 1). This result is in agreement with previous studies ${ }^{9,10}$ and was clearly due to the origin of the examined samples as they were obtained from AD systems producing methane. It is well known that the archaeal species present a narrow diversity in the biogas microbiome. The outcomes of the present research demonstrate that the high transcriptional activity of the methanogens is the main determinant which justifies their efficient role in the conversion of all intermediate metabolites produced by the diverse bacterial consortium. Transcripts encoding enzymes for other basal functioning of the cellular machinery, such as protein biosynthesis (ribosome in Figure 2), protein folding, transcription and ATP synthases, were also found to have high abundance. Moreover, genes belonging to the "fatty acids" category were also highly expressed. Nevertheless, at this level of analysis, the differences in the transcriptional activation of the microbes before and after the LCFA addition could not be evidenced. The inability to discriminate the influence of the LCFA on the microbial activity was probably due to the large number of genes grouped in this general functional category. However, as it will be further discussed, the metatranscriptomic analysis on single genes revealed that the fatty acid degradation pathway was one of the most deeply influenced due to the modifications of the feedstock composition.

Another interesting finding was related to the presence of pathogens in the biogas reactors. In contrary with a recent study, which did not indicate the presence of putative pathogenic microorganisms in the biogas plants ${ }^{36}$, the current metatranscriptomic analysis identified highly expressed gene categories related to pathogenicity islands (Figure 1). For applicability reasons, this finding is of a major importance considering that the digested effluent from the biogas plants is commonly utilized as soil fertilizer. The presence of pathogenic activity in the digestate raises risks and concerns for the health of living organisms in the area 
around the effluent disposal. Thus, a further investigation is needed in order to deeply characterize the role of these putative pathogenic genes.

Similar findings were obtained from the analysis performed at a higher level of functional detail using "KO$3^{\text {rd }}$ level". Methane metabolism and major metabolic pathways for the microbial survival were among the most abundant categories. Nevertheless, it was remarkably found that genes belonging to some specific pathways relevant to the $\mathrm{AD}$ process, such as "butanoate" and "propanoate" metabolisms, have on average a low transcriptional level.

Deeper insights in the functional dynamics were gained by performing the "KEGG-orthology functions" analysis (Dataset S2), in which the transcriptome of each biogas reactor (biological replicate) was compared prior and after the LCFA addition. The main advantage of this analysis relies on its specificity targeting single gene functions, in contrary with the previous analyses that provided a global functional overview. It should be noted that the findings reported for a certain gene function could be an outcome driven by different microbial species. The results showed that a large part of the more expressed genes (absolute expression level) were related to methanogenesis. More specifically, such genes are the ones encoding the subunits of the methyl-coenzyme $\mathrm{M}$ reductase $(m c r B, m c r A, m c r C, m c r G, m c r D)$ which catalyzes the final step in methanogenesis, $m t d$ (methylenetetrahydromethanopterin dehydrogenase) which is involved in the formation of methane from $\mathrm{CO}_{2}$, or subunits of the tetrahydromethanopterin S-methyltransferase ( $m t r H, m t r E, m t r D$, $m t r C, m t r F$ ) which is involved in the formation of methane from $\mathrm{CO}$. The preferable hydrogenotrophic pathway in the current experiment was a direct effect of the high abundance of the methanogen Methanoculleus sp. DTU006 ${ }^{5}$. The relevance of the methanogenic pathway from a transcriptional point of view is also evidenced by reporting the expression levels of the genes identified in the assembly on the "global" KEGG pathway map, and more specifically on the methane pathway (KO00680) (Supporting Information, Figure S1).

Other highly expressed KEGG-orthology functions were related to genes encoding proteins with basal roles, such as flagellin fliC, cell division protein FtsZ, and molecular chaperone DnaK. Moreover, genes related to lipid degradation exhibited a medium/low expression, even after the LCFA addition. Nine genes involved in fatty acid degradation were expressed (out of 30 reported in KEGG) (path:map00071). Notably, these genes 
were mainly encoding various dehydrogenases such as $a d h E$ (EC:1.2.1.10 1.1.1.1), HADH (EC:1.1.1.35), E1.2.1.3 (EC:1.2.1.3), DCAA (EC:1.3.99.-), fadB (EC:1.1.1.35 4.2.1.17 5.1.2.3 5.3.3.8) and fadJ

(EC:1.1.1.35 4.2.1.17 5.1.2.3), ferredoxin--NAD+ reductase $h c a D$ (EC:1.18.1.3) and acetyl-CoA acyltransferase 1 ACAAl (EC:2.3.1.16). However, none of these genes was clearly up-regulated in all the three replicates in response to LCFA addition.

The results from the present study clearly prove that the shotgun metagenomics reads efficiently describe the global functional characteristics of the AD microbial community (Dataset S2). Nevertheless, their efficiency in monitoring the most relevant transcriptional modifications induced by the addition of LCFA is found to be limited. The apparently stable gene expression level detected can be mainly attributed to the fact that the transcriptomic analysis in meta-samples does not target genes of single species. Thus the gene functions of many species are merged together and are provided as a unique result. This is due to the impossibility of assigning with high level of confidence the RNA reads to the genes of the single species using the MGRAST software. Since it is expected that orthologous genes in different species present a variable response to a given perturbation, the final result can be blurry and the transcriptional level for most of the genes is unchanged. To solve this conundrum, an additional analysis was performed by aligning the RNA-seq reads to single genes extracted from the metagenome assembly.

\section{Global effect of LCFA on the transcriptional level of the GBs extracted from the biogas metagenome} assembly.

In order to interpret the results of this analysis, the functional behavior of the microbial community was described using two terms; a) the "absolute expression level", which is defined as the total number of RNA reads assigned to each microorganism and b) the "transcriptional activity", which is the ratio between the RNA reads to the DNA reads for each microbe. This resulted in three outcomes which are the identification of the most active species in the microbial community, the investigation of specific microbes that become less active even if they maintain steady their relative abundance, and finally the monitoring of the species behavior in response to LCFA exposure. Taxonomic, genomic and functional characteristics of the species included in the present analysis were previously described by Campanaro and coll. ${ }^{5}$ (Additional File 3, Table S2) and in the present paper in Dataset S1. 
The absolute expression level of the microbes after LCFA addition impressively varied over six orders of magnitude and ranged from less than 0.0001 to 137 (Figure 3, Dataset S4). Moreover, the outcomes from the transcriptional activity revealed a direct correlation between the abundance of the majority of the GBs and their absolute expression level. This means that the more dominant a certain microbe is, the higher its absolute expression level will be, and vice versa. More specifically, regarding the bacterial community, Pr06 (Alcaligenaceae sp. DTU102), Fi63 (Eubacteriaceae sp. DTU096), Ba02 (Rikenellaceae sp. DTU002), Fi57 (Clostridiales sp. DTU089) and Ba05 (Porphyromonadaceae sp. DTU047) were the most abundant GBs and thus presented the higher expression level after the addition of LCFA in the feedstock. It was remarkable that none of these species were related to fatty-acid oxidizing bacteria. This suggests that known fatty-acids oxidizers, such as species belonging to Syntrophomonadaceae and Syntrophobacteraceae families, ${ }^{14-17,37}$ do not dominate the microbial community, even in the present case that the biogas reactor was processing lipid rich substrate. Nevertheless, as it will be further discussed, the genes of Syntrophomonas sp. were strongly up-regulated in response to the LCFA addition confirming their important role in the degradation of such compounds.

Transcripts related to methanogenesis were found to be predominant in our analysis. This was attributed mainly to the prevalence of Methanoculleus sp. DTU006 (Eu01) and to the high expression value of its genes involved in methanogenesis (Figure 3 and Supporting Information, Figure S1b). Methanosarcina sp. DTU009 (Eu04) increased proportionally both its abundance and its transcriptional activity after the LCFA exposure, Methanothermobacter sp. DTU051 (Eu05) has a slight increase and the other methanogens remained substantially stable. In contrast, only the Euryarchaeota DTU008 (Eu03) slightly decreased in abundance and thus its absolute expression level, showing a negative influence caused by LCFA. Noticeably, $\mathrm{Eu} 01, \mathrm{Eu} 02$ and $\mathrm{Eu} 03$ were classified in the group of $15 \mathrm{GBs}$ demonstrating the highest expression level in the microbial community after LCFA addition.

Since in the cited literature there is not any information concerning the identification of the most active fraction of the biogas microbial community, the current analysis of the transcriptional activity provided deep insights to elucidate this aspect. Previous studies in relevant scientific fields reported contradictory findings; Leimena et al., ${ }^{38}$ found a substantial similarity between results obtained from the metatranscriptome and the 
metagenome of gut microbiome, while other investigations on the same topic showed the presence of a specific fraction of species that are inactive ${ }^{8,39}$. Our analysis showed that the entire microbial community is active and thus the presence of GBs with an extremely low transcriptional activity was not evidenced (Figures 3 and 4).

Comparative analysis of species abundance and "absolute expression level" revealed three different groups of microbes responding differently to LCFA addition. Part of the microbial community remained unchanged in both these two parameters; these GBs (e.g. Fi55, Fi01) are represented in the central section of Figure 4. A second group increased (e.g. Fi23, Fi25) or decreased (e.g. Sy06, Fi12) concertedly both in terms of abundance and "absolute expression level" and they are close to the diagonal line in Figure 4 (but far from the central section). This behavior suggests that in some species the change in abundance is the main responsible for the modification of the "absolute expression level". The third group (e.g. Fi26, Ba02) included GBs where these two parameters did not change concertedly and diverged from the diagonal line in Figure 4. In some of these GBs (e.g. Fi26, Fi27 and Pr11) the abundance increased more than their absolute expression level, while in others (e.g. Ba02, Fi57, Fi66, Fi67, Fi68, Pr02 and Pr06) the situation is reversed. In the third group of GBs the ratio between RNA reads and DNA reads is modified by the exposure to LCFA, and this outcome is interesting because it evidenced a modification of the "transcriptional activity". The different behaviors evidenced revealed an extremely variable effect of LCFA on the microbial species.

The strong modification of the microbial consortium after the LCFA exposure hampers the identification of differentially expressed genes. This is due to the fact that both the species' transcriptional regulation and their relative abundance are changing concurrently contributing in the determination of the final result. Frequently, in metatranscriptomics this combined effect cannot be discriminated as the variation of the microbial abundance is unknown. The obtained metagenomic and metatranscriptomic information gives the privilege to the current study to be able to identify the influence of each factor to the final result. Therefore, the genes that were found to be statistically differentially expressed in the RNA-seq analysis were compared with the corresponding ones obtained from the same statistical procedure applied to the DNA-reads. (see materials and methods). This procedure led to the discrimination of the changes driven by the genome abundance from those attributed to the transcriptional effect of LCFA on gene regulation (Figure 5). 
The GBs with the higher number of differentially expressed genes (up or down-regulated) are reported in Figure 5. The analysis demonstrated that Syntrophomonas sp. (Fi08 and Fi10) presented an obvious upregulation of their transcriptional profile. Previous studies associated the degradation of fatty acid compounds with Syntrophomonas species ${ }^{13,40}$. Moreover, it was shown that LCFA led to an up-regulation of transcripts encoded in Methanoculleus species (Eu01, Eu02) (Figure 5). Surprisingly, as it will be further discussed these over expressed genes were not functionally related despite that both methanogens belong to the same genus. Another interesting outcome was that among the GBs with the higher number of differentially expressed genes, only four of them were found to change in abundance more than two folds after the LCFA addition (i.e. Fi10, Fi08 and Fi09 increased and Fi07 decreased). Nevertheless, it was demonstrated that the fatty acids influenced indirectly many other species inside the microbiome even if their abundance remained stable. This indicates that the transcriptional activation of some genes allows the microorganisms to counteract the toxic effect of fatty acids, providing a "protective mechanism". This can be clarified by analyzing the functional annotation of the genes as it will be described in the next section.

\section{Expression analysis of the genes included in the metagenome assembly.}

By shedding the light on the functional roles of the differentially expressed genes identified, it is possible (a) to acquire new insights on the inhibitory effect of LCFA to the microbial community dynamicity, (b) to identify which genes are involved in fatty acids degradation and (c) to determine the protective mechanisms implemented by the bacterial species towards the LCFA toxicity.

The current analysis identified 51,469 expressed genes (having at least one sequence from the RNA-seq experiment) (Dataset S3), 1,254 of them were found differentially expressed using edgeR software (Dataset S3). By further refining the results, 234 genes were discarded as their difference in expression was attributed to changes in the GB abundance. The remaining 1,020 differentially expressed genes were grouped considering their COG category and their functional role defined according to the KEGG metabolic pathways (Figure 6).

COG analysis verified the strong influence of the LCFA on the microbial community (Figure 6a) as the genes that were found to be up- or down-regulated were classified to a wide range of different functional categories. An interesting result was related with the class C "Energy production and conversion", in which 
numerous genes were down-regulated. Nevertheless, the methane metabolism, which is the most crucial energy conversion process in the studied AD system, included mainly up-regulated genes (KEGG analysis, Figure $6 b$ ). A better representation could be obtained from the construction of the methane pathway map (Supporting Information, Figure S1), which shows the transcriptional change of individual genes. It is clearly demonstrated that the preferable pathway during the degradation of unsaturated fatty acids is hydrogenotrophic methanogenesis. This is evidenced by the up-regulated genes involved in the methanogenesis, most of them are involved in the hydrogenotrophic pathway (e.g. $m c r, h d r, m t r A, f w d A$, $m t d, f r h A$; see Supporting Information, Figure S1), while only one is involved in the acetoclastic pathway.

Moreover, the up-regulated genes in the methane pathway belong mainly to Eu02 (Methanoculleus sp. DTU007) which surprisingly has a lower relative abundance compared to Eu01 (Methanoculleus sp. DTU006) (Figure 4). As previously discussed, the over expressed genes of these Methanoculleus sp. were not functionally related despite that both methanogens belong to the same genus. Most of the differentially expressed genes of Eu01 could not be assigned to KEGG pathways, and therefore are functionally unknown, while from the assigned ones the most abundant group belongs to ABC-type transporters (e.g. molybdate transport system, periplasmic component, spermidine/putrescine transport systems etc.) (Dataset S3). Till now, it was only known that the methanogens have different tolerance level towards LCFA inhibition and more specifically that hydrogenotrophic methanogens are more resilient compared to the acetoclastic ones ${ }^{41}$. The current research additionally proved that LCFA influence differently the hydrogenotrophic archaea even if they belong to the same genus (i.e. Methanoculleus).

An interesting pathway which is related with hydrogenontrophic methanogenesis is the Syntrophic Acetate Oxidation-SAO (i.e. reversed Wood-Ljungdahl Pathway, WLP). The mechanism of this pathway is that acetate is converted to hydrogen and carbon dioxide by SAO bacteria ${ }^{42-44}$. These resulting compounds are then passed to methanogens resulting in a reaction thermodynamically driven by the $\mathrm{H}_{2}$ consumption of hydrogenotrophic methanogens generating methane ${ }^{45,46}$ Analysis of the genes belonging to the WLP (KEGG entry M00377) showed the presence of 121 expressed genes (having at least one sequence read). Out of these genes, 91 were assigned to specific GBs revealing that Clostridia sp. DTU068 (Fi38) and Clostridia sp. DTU095 (Fi62) have a nearly-complete expressed pathway (Supporting Information, Figure S1). 
Moreover, the taxonomic assignment of Fi38 ${ }^{5}$ suggested a phylogenetic correlation with Thermacetogenium phaeum, an anaerobic, thermophilic, SAOB isolated from a thermophilic anaerobic methanogenic reactor ${ }^{47}$ The high expression level of transcripts involved in WLP for Fi38 is additional information that increases the probability of correct taxonomic assignment and verifies at least that this specific GB is a SAOB.

Considering the genes belonging to the fatty acid degradation pathway, the result showed that five genes were up-regulated. Interestingly, four genes belong to Syntrophomonas sp., (Fi08-Syntrophomonas sp. DTU017 and Fi10-Syntrophomonas sp. DTU019) and one cannot be assigned to any GB (Dataset S3 and Supporting Information, Figure S1). The findings confirm the association of Syntrophomonas species in the degradation of the LCFA and also validate the correctness of the previous taxonomical assignment of these GBs ${ }^{5}$.

It is also noteworthy that genes involved in "peptidoglycan biosynthesis" and in "lipopolysaccharides biosynthesis" are up-regulated (Figure 6) suggesting that the microbes are protected from the toxic effect of LCFA by modifying their cell wall and the composition of the lipopolysaccharides (LPS). A putative protective role for the external structures of the cell has been previously proposed. Some bacteria increase their cell-wall thickness resulting in an increment in affinity of peptidoglycan layers for specific molecules and thus they become more resistant to toxic compounds ${ }^{48-51}$. Moreover, LPS is the major component of the outer membrane of Gram-negative bacteria and it can contribute in protecting the membrane from specific chemical compounds, such as fatty acids ${ }^{52}$. For this reason it is possible that a modification of its structure can counteract the inhibitory effect of LCFA. Notably these genes are not encoded by species associated to the Synthrophomonas genus but to some GBs belonging to the families Clostridiales (Fi04, Fi20, Fi22, Fi30, Fi49), Rykenellaceae (Ba01), or to the genera Halothermothrix (Fi21) and Anaerobaculum (Sy01). All these species (apart from Sy01 that slightly decrease its abundance) remained stable after LCFA addition suggesting that the protective mechanism does not confer a strong advantage towards the rest microbial community but has mainly an "homeostatic role".

It was previously demonstrated that unsaturated LCFAs (e.g. oleic acid) are enabling the activation of twitching-mediated chemotaxis for some species, such as Pseudomonas aeruginosa ${ }^{53}$. This means that some bacteria are able to perceive fatty acids and to migrate up a gradient of these compounds. Two of the KEGG 
classes identified in this study, having the higher number of differentially expressed genes, are the "twocomponent system" and "bacterial chemotaxis" (Figure 6). The genes belonging to these classes encode flagellins like fliC but, more importantly, proteins of the chemotaxis complex like CheW, CheA and MPC (methyl-accepting chemotaxis protein). The signal transduction protein CheW-like is part of the chemotaxis complex of the hydrocarbon degrading bacteria Alcanivorax dieselolei. The encoding gene of CheW-like can be induced in response to the presence of long-chain alkanes ${ }^{54}$ and this indicated that the chemotactic response is modulated at a transcriptional level. Moreover, CheW has an essential role in the chemotaxis signal transduction in numerous bacteria ${ }^{55-60}$. CheW couples MCP to the histidine kinase CheA ${ }^{61}$ and our findings indicate that the transcriptional regulation of these three proteins is a key essential point for the chemotactic activity of some bacterial species in presence of LCFA. Curiously, the GBs showing transcriptional up-regulation of these genes are not related to the Syntrophomonas and Syntrophothermus genera, but belong to Clostridia class (Fi20, Fi22, Fi30, Fi36, Fi37), to Halothermothrix (Fi21) and to Tepidanaerobacter (Fi34). Despite this "chemotactic activity", only Fi37 increased its abundance in response to LCFA addition (Figure 4) suggesting that these species are not strongly favored by the unsaturated fatty acid environment. The incomplete fatty acid degradation pathway of the GBs presenting "chemotactic activity" suggests that these species are unable to use 18 carbon unsaturated fatty acid in a canonical $\beta$ oxidation process. Therefore, possible explanations are that they are able to utilize subproducts derived from oleate degradation performed by Syntrophomonas species or that they cannot degrade unsaturated fatty acids, but rather saturated ones, even if they enable "chemotactic activity". Since in the cited literature the metatranscriptome of biogas reactors is partially explored and due to the fact that the majority of the GBs are recently identified, a rational beyond this phenomenon can only be hypothesized, and further investigation is definitely needed.

This study represents a new point of view in the metatranscriptomic analysis of the biogas microbial community. The dissection of the transcriptomic effect of unsaturated LCFA at a species-specific level allows a better comprehension of the effects induced by this compound. Thus, it was possible to gain new insights concerning the functional role of single microbial species in the biogas community. The findings highlight that, apart from the role of Synthrophomonas species in fatty acid utilization, the resistance level of the microbes to LCFA inhibition is extremely important. It was also concluded that the biogas production 
process is executed by a very complex and integrated pattern of functions shaped by the specialization of the GBs in microbial consortium. It was evident that the presence of an additional energy source (i.e. LCFA), together with the inhibitory effect of fatty acids on the microbial species, definitely perturbed deeply the equilibrium of the microbiome. By modifying the transcriptional activity, the microbial cells can establish protecting mechanisms in order to maintain a proper functionality into the AD system. Additionally, specific microbes enable the chemotactic response once fatty acids are present in the biogas reactor. The microbial adaptation to LCFA had a high importance in some species of the microbial community and is strongly determined by a deep modification of their transcriptional activity. The current work can be a pilot for further metatranscriptomic studies related to the biogas microbiome.

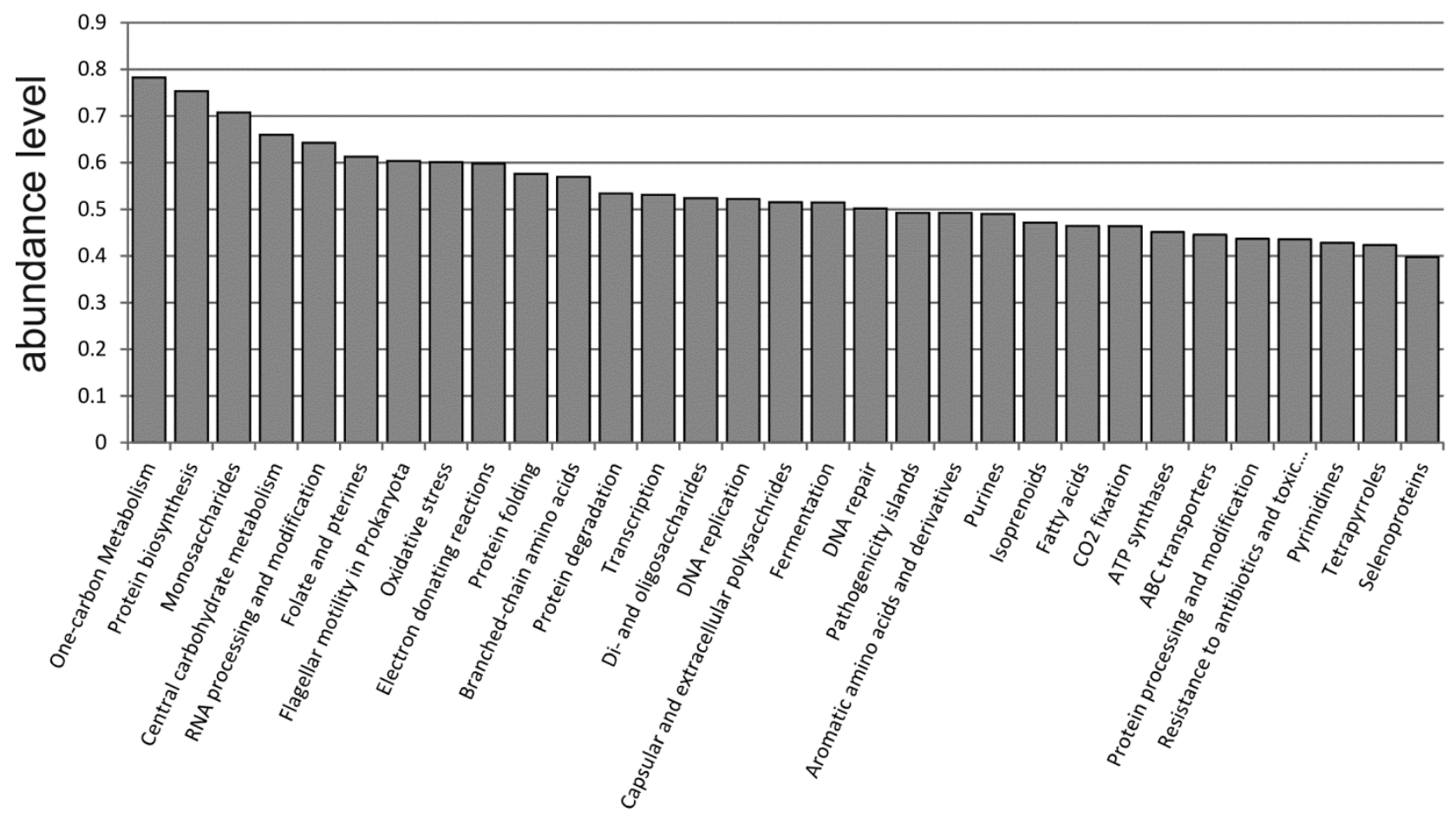

Figure 1: Average abundance level of the genes in the six experimental samples examined. RNA-seq reads were assigned to different functional classes using "subsystem- 2 nd level" in MGRAST database. The data are not considered separately for prior and after LCFA addition because the differences in expression were negligible. Only the functional classes with the higher expression are reported. 


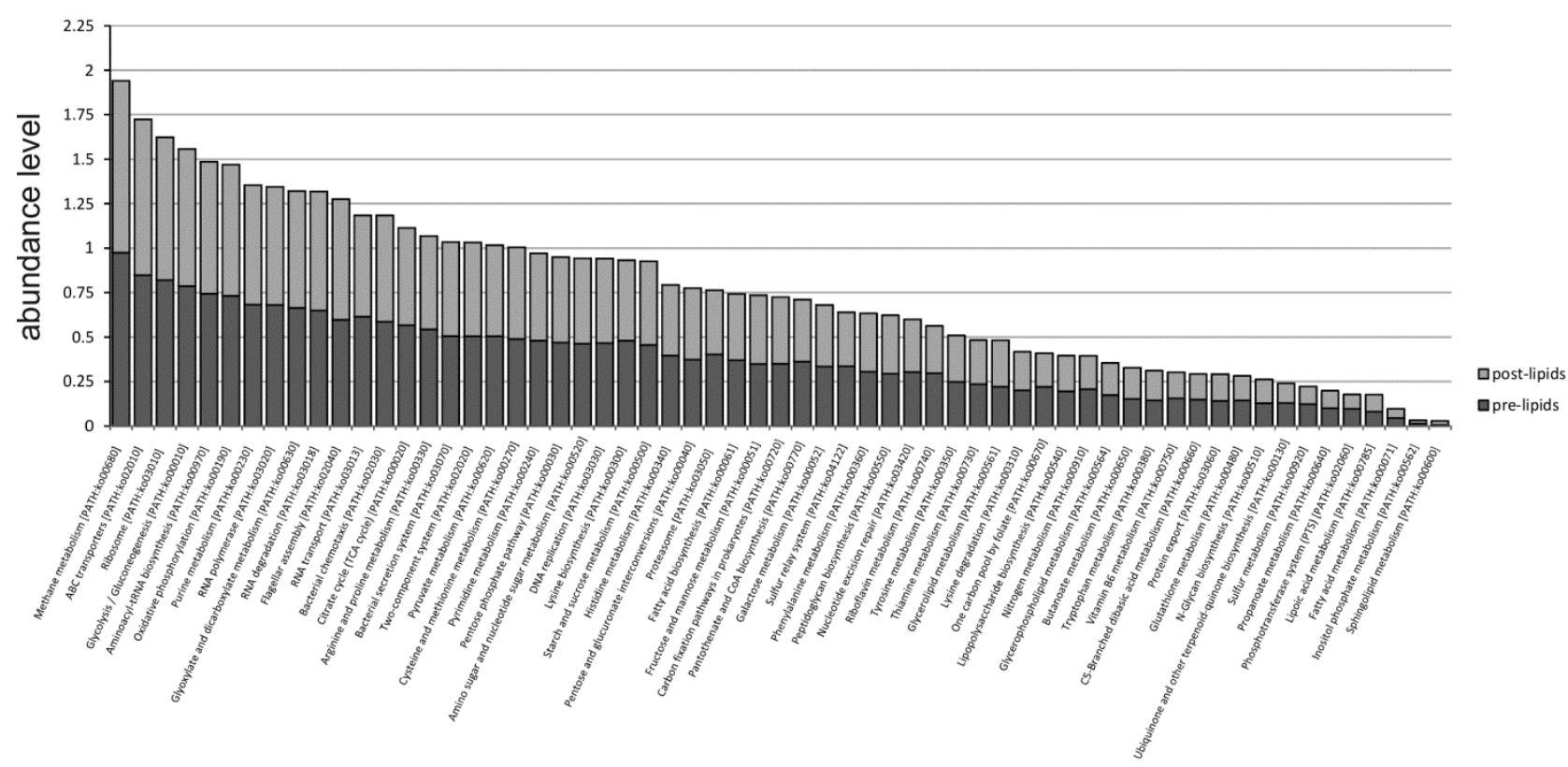

Figure 2: Average abundance level of the genes assigned to different functional classes using "KO $3{ }^{\text {rd }}$ level" in MGRAST database. Data are reported as stacked columns reporting values obtained prior and after lipid addition in the bottom and top part of each column. Only the more represented functional activities are reported in figure, while categories related to eukaryotic-specific processes and to degradation of xenobiotic compounds were excluded from the representation. Results obtained prior and after the LCFA addition are represented in figure with dark and light grey respectively. 


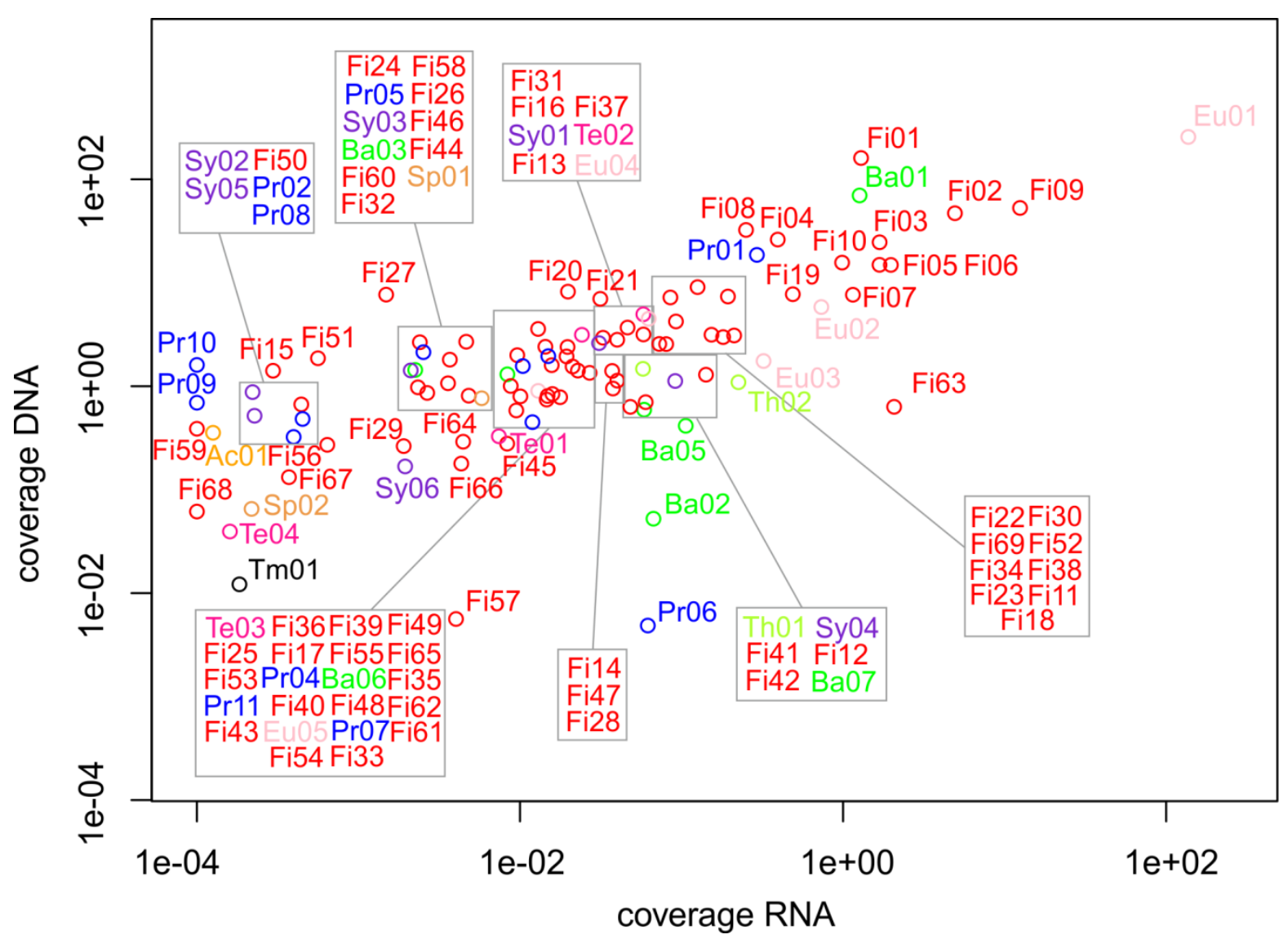

Figure 3: Comparison between the coverage of the GBs calculated using the DNA sequences (species abundance) and RNA sequences (average transcriptional activity). Data refer to the average value determined for the three replicates obtained after LCFA addition. Colors were assigned considering the phylum assigned to each GB. Actinobacteria (orange), Bacteroidetes (green), Euryarchaeota (pink), Firmicutes (red), Tenericutes (magenta), Proteobacteria (blue), Synergistetes (violet), Thermotogae (acid green), Spirochaete (light brown) and TM7 (black). Axes are in logarithmic scale to facilitate visualization of the values that are spread in a wide range, indicating strongly different expression activity for the different GBs. 


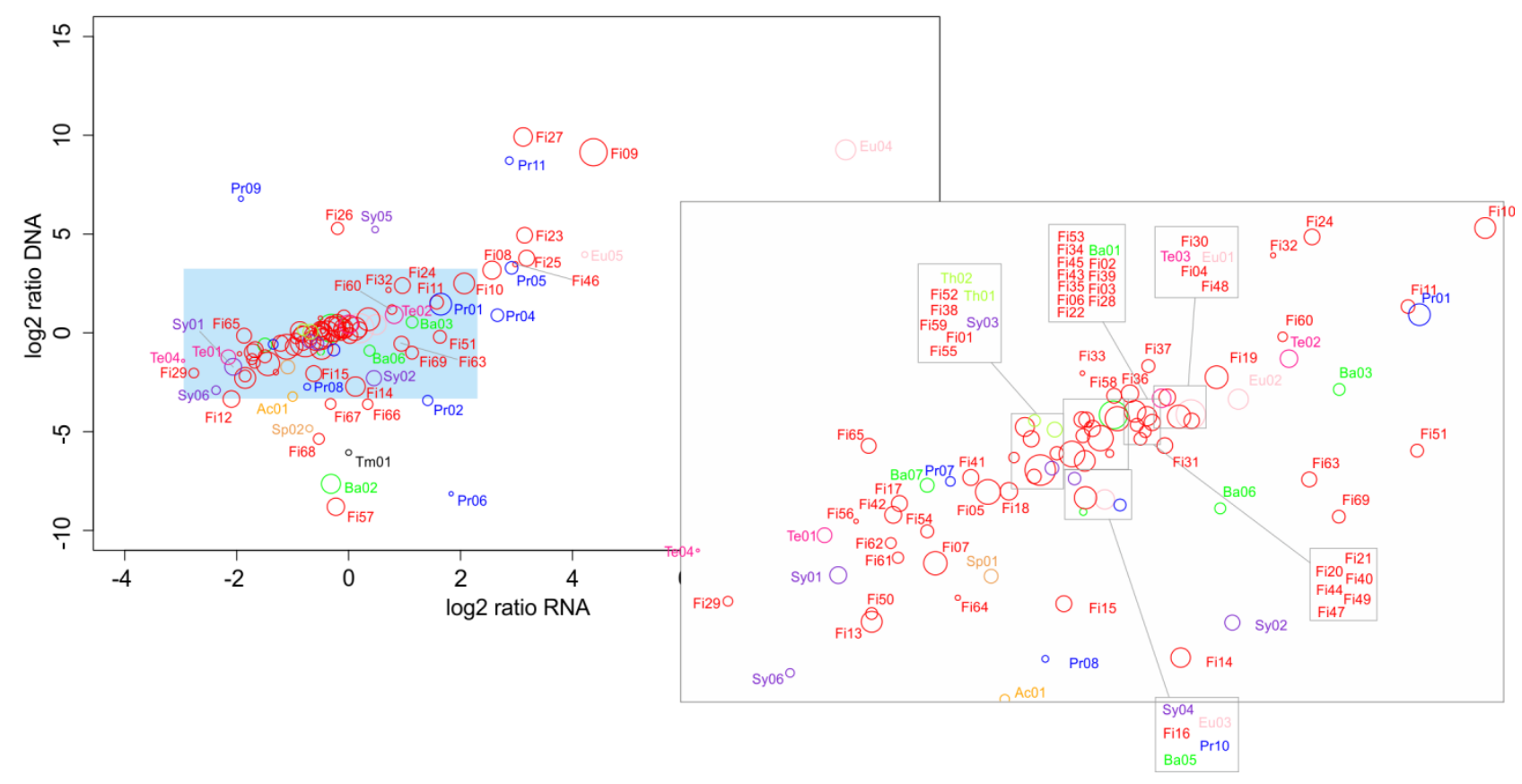

Figure 4: Ratios calculated considering post vs. pre LCFA addition for the DNA coverage (Y axes) and RNA coverage ( $\mathrm{X}$ axes). The plot compares variations in species abundance ( $\mathrm{Y}$ axes) with variations in absolute expression level ( $\mathrm{X}$ axes) prior and after LCFA addition. The central section (light blue rectangle) is enlarged on the right part of the figure. Colors of the circles are proportional (logarithmic scale) to the average coverage of the GBs (DNA coverage) across all the six experiments analyzed (three "pre" and three “post-LCFA" addition). GBs having positive values both for DNA and RNA coverage ratios (i.e. Eu05) increased after lipid addition both considering genome abundance and transcriptional level, those having negative values (i.e. Sy06) decreased in both. 


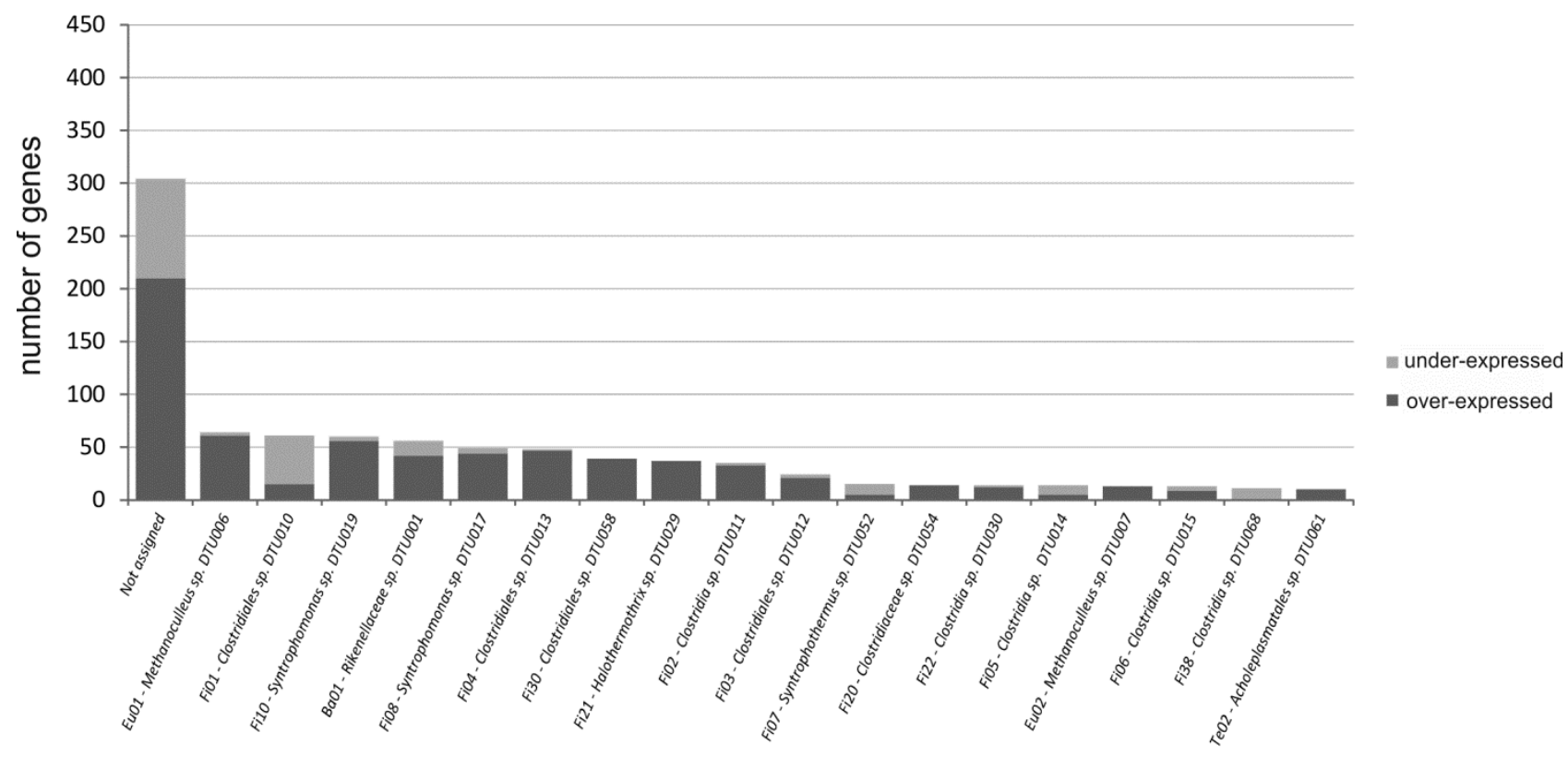

Figure 5: Number of differentially expressed genes identified for each GB. The number of differentially expressed genes identified for each GB and those that cannot be assigned to binned scaffolds (not assigned) is shown. The results are "normalized" by taking into account the variation in genome abundance. This "normalization" process improved the identification of differentially expressed genes by "subtracting" the effect determined by changes in genome abundance. 


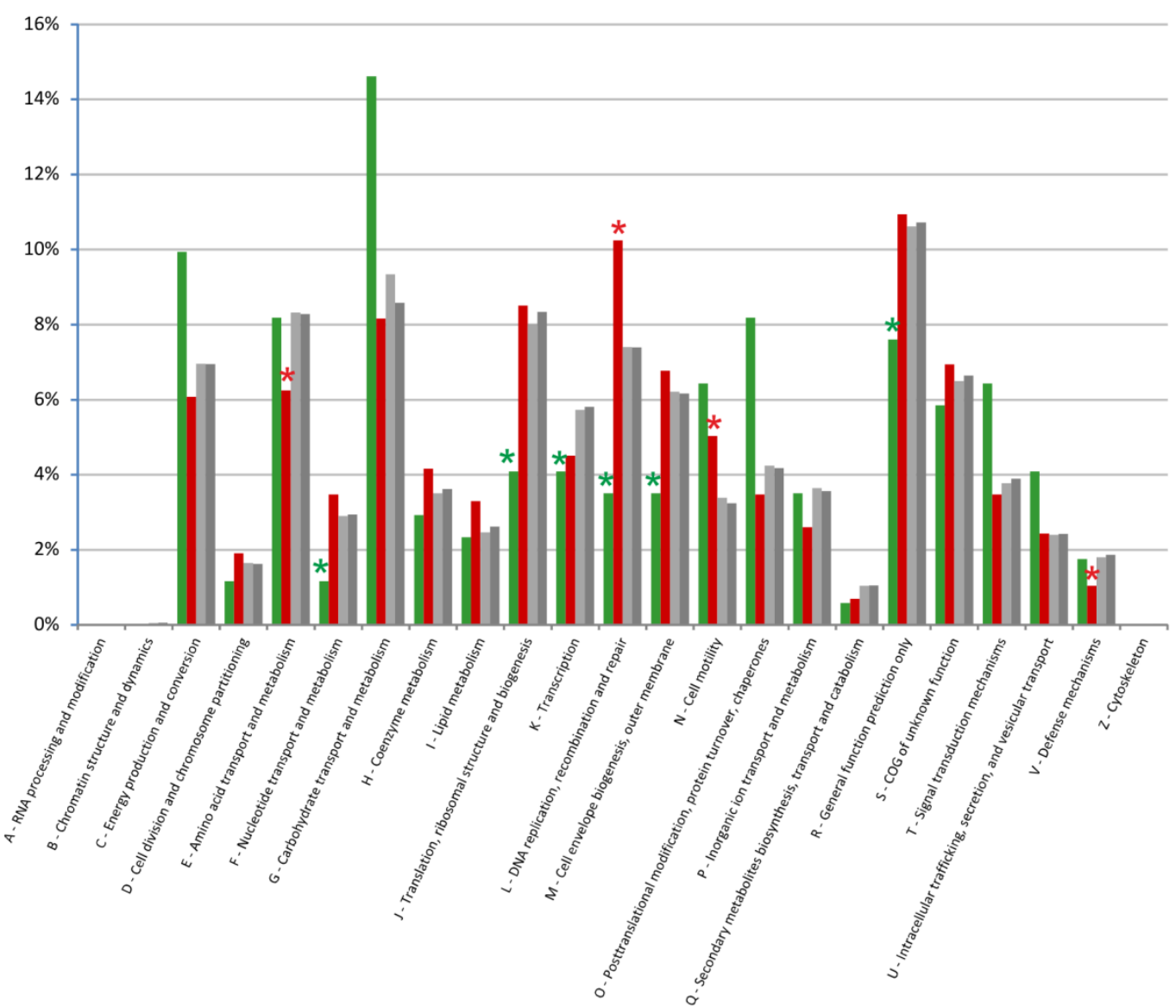

- under-expressed

- over-expressed

= COG total pre-lipid

n COG total post-lipid

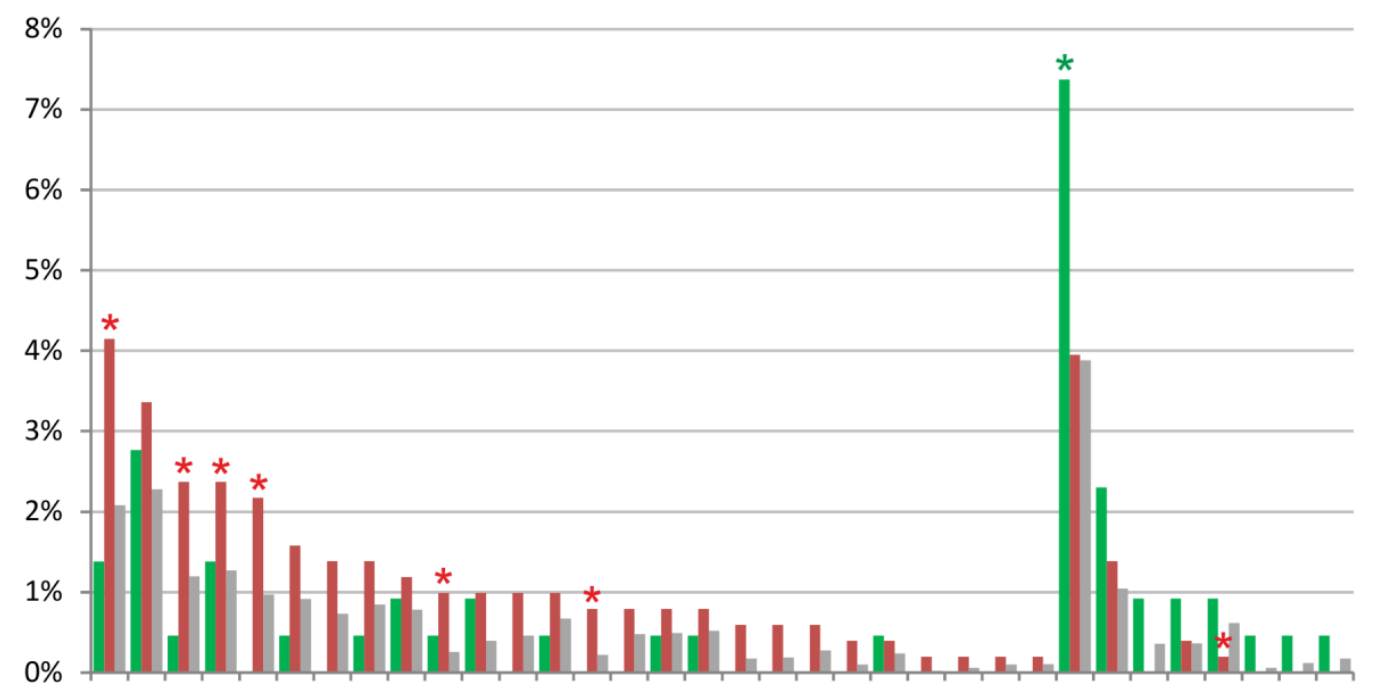

— under-expressed

- over-expressed

= KEGG total

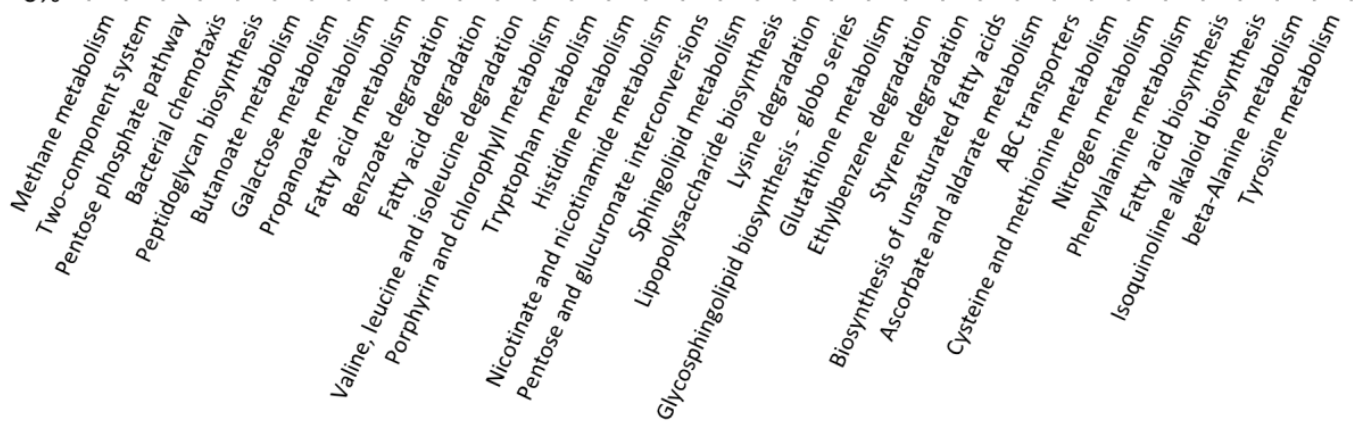


Figure 6: Functional classes of the differentially expressed genes. (a) COG analysis of the over/underexpressed genes. Fraction (\%) of the differentially expressed genes assigned to different COG categories and comparison with the COG percentage determined for all the expressed genes (having at least one read) in the entire assembly. Functional classes marked with $(*)$ are those statistically enriched with differentially expressed genes. (b) The same analysis is reported considering assignment of the differentially expressed genes to the KEGG categories.

\section{TOC/ABSTRACT ART}

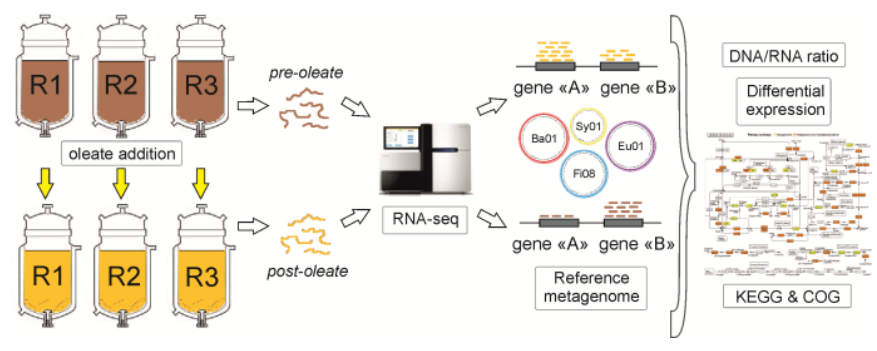

\section{ASSOCIATED CONTENT}

Supporting Information. Reactors operational performance (Table S1); representation of the gene expression level in the context of the KEGG metabolic pathways (Figure S1); RNA-seq reads mapped to some selected regions of Eu01 (Figure S2); Agilent results (Figure S3); comparison between technical replicates (Figure S4). Results obtained for analysis of gene expression are reported in Datasets S1-S3. This material is available free of charge via the Internet at http://pubs.acs.org.

Dataset S1: Correspondence between acronyms used for the GBs and the complete name proposed. In columns are reported: (A) GB acronym, (B) extended name proposed for the GB, (C) domain (Archaea or Bacteria), (D) phylum, (E) taxonomic level considered for the assignment of the extended name, (F) estimated level of completeness , (G) estimated level of contamination. Results reported in (F) and (G) were from a previous study ${ }^{5}$. 
Dataset S2: Table reporting the gene functions determined according to "subsystem 2nd level", "KEGG orthology 3rd level" and "KEGG orthology function level" (column A) and their expression levels (columns B-G) obtained analyzing the RNA random sequences in the MG-RAST database. Results reported were obtained for the three replicates obtained before (columns B. C, D) and after (columns E, F, G) LCFA addition. $\log 2$ ratios obtained comparing expression level "after-LCFA" and "before-LCFA" addition for each biological replicate are reported in columns H, I, J. The average level of expression before-LCFA is reported in column $(\mathrm{K})$, its standard deviation in column $(\mathrm{L})$, the average level of expression after-LCFA is reported in column $(\mathrm{M})$, its standard deviation in column $(\mathrm{N})$.

Dataset S3: Table reporting the differentially expressed genes identified (worksheet "DE genes") and the number of reads aligned pre gene determined in the RNA-seq experiment (worksheet "expressed genes").

DE genes worksheet: (A) gene ID, (B) assignment of the genes to the GBs, (C) COG annotation, (D) COG functional category, (E) gene name according to COG annotation, (F) gene function according to COG annotation, (G) KEGG ID, (H) gene name according to KEGG, (I) KEGG pathway, (J) ratio of expression values (RNA-seq experiment - post vs. pre LCFA addition) calculated using edgeR, (K) ratio of coverage values (DNA-seq experiment - post vs. pre LCFA addition) calculated using edgeR, (L) ratio of the expression values calculated after removing the contribution derived from column " $K$ ", (M) p-value determined using edgeR from the biological replicates of the RNA-seq experiments, $(\mathrm{N}) \mathrm{p}$-value determined using edgeR from the biological replicates of the DNA-seq experiments. Data reported in columns (A-I) and (K-N) were from ${ }^{5}$. Genes differentially expressed were determined using edgeR software on the RNA-seq data (column "J") and were normalized considering the results obtained on the genome ratio (column "K") using the same software. Cells highlighted in grey in column " $\mathrm{L}$ " refer to genes that were not considered as differentially expressed after removing the contribution due to changes in genome abundance (column " $K$ "). Cells highlighted in grey in columns "A-I" are those where the ratio for genome abundance was not determined by edgeR (after normalization); for these data the genome ratio was directly calculated from row data and the p-value for genome ratio determined by edgeR software was not reported ("ND" in column "K". KEGG codes extracted from these results (only for up/down-regulated genes more than two-folds) were used to assign colors to the genes in the KEGG pathways (Supporting Information, Figure S1). Expressed genes worksheet: data reported in columns "A-I" are the same reported in "DE genes worksheet", (J-L) number of 
RNA-seq reads aligned on genes determined in the genome assembly before LCFA addition in three biological replicates, (M-O) number of RNA-seq reads aligned on genes determined in the genome assembly after LCFA addition in three biological replicates.

Dataset S4: Table reporting the coverage determined for the GBs considering RNA-seq (present experiment) and metagenomics sequencing (data from Campanaro $S$ and coll. 2016). In columns are reported: (A) GB acronym, (B) extended name proposed for the GB, (C-H) coverage values determined from RNA-seq data obtained for the six experiments, (I) average value for RNA-seq coverage before-LCFA, (J) average value for RNA-seq coverage after-LCFA, (K-P) coverage values determined from metagenomic (DNAsequencing) data in the same six experiments, (Q) average value for metagenomic coverage before-LCFA, (J) average value for metagenomic coverage after-LCFA.

\section{AUTHOR INFORMATION}

\section{Corresponding Author}

* Department of Biology, University of Padova, Via U. Bassi 58/b, 35121, Padova (Italy); e-mail: stefano.campanaro@unipd.it ; phone: +39 0498276306; fax: +39 0498276230

\section{Author Contributions}

\# These authors contributed equally to the work.

L.T. monitored bioreactors performance, collected the samples and prepared RNA for sequencing, analyzed data and revised the manuscript; S.C. designed the strategy for metatranscriptomics data analysis, analyzed the data and wrote the manuscript; P.G.K. designed experiments, setup bioreactors, monitored bioreactors performance, and wrote the manuscript; X.Z. setup bioreactors, monitored bioreactors performance; I.A. designed and supervised experiments, wrote the manuscript. All authors have given approval to the final version of the manuscript. 


\section{ACKNOWLEDGMENT}

This work was supported by the Danish Council for Strategic Research under the project "SYMBIO Integration of biomass and wind power for biogas enhancement and upgrading via hydrogen assisted anaerobic digestion", contract 12-132654. Quality control of RNA samples and Illumina sequencing was performed at the Ramaciotti Centre for Genomics (Sydney, Australia).

\section{ABBREVIATIONS}

AD, anaerobic digestion; LCFA, Long Chain Fatty Acids; CSTR, continuous stirred tank reactors; CM, cattle manure; GB, genome bin; KO, KEGG-orthology; LPS, Lipopolysaccharides.

\section{REFERENCES}

1. Riviere, D.; Desvignes, V.; Pelletier, E.; Chaussonnerie, S.; Guermazi, S.; Weissenbach, J.; Li, T.; Camacho, P.; Sghir, A. Towards the definition of a core of microorganisms involved in anaerobic digestion of sludge. The ISME journal 2009, 3 (6), 700-714.

2. Werner, J.J.; Knights, D.; Garcia, M.L.; Scalfone, N.B.; Smith, S.; Yarasheski, K.; Cummings, T.A.; Beers, A.R.; Knight, R.; Angenent, L.T. Bacterial community structures are unique and resilient in full-scale bioenergy systems. Proc. Natl. Acad. Sci. U. S. A. 2011, 108 (10), 4158-4163; 10.1073/pnas.1015676108 [doi].

3. De Francisci, D.; Kougias, P.G.; Treu, L.; Campanaro, S.; Angelidaki, I. Microbial diversity and dynamicity of biogas reactors due to radical changes of feedstock composition. Bioresour. Technol. 2015, 176, 56-64; 10.1016/j.biortech.2014.10.126 [doi]. 
4. Nobu, M.K.; Narihiro, T.; Rinke, C.; Kamagata, Y.; Tringe, S.G.; Woyke, T.; Liu, W. Microbial dark matter ecogenomics reveals complex synergistic networks in a methanogenic bioreactor. The ISME journal 2015, 9 (8),1710-1722; 10.1038/ismej.2014.256 [doi].

5. Campanaro, S.; Treu, L.; Kougias, P.G.; De Francisci, D.; Angelidaki, I. Metagenomic analysis and functional characterization of the biogas microbiome using high throughput shotgun sequencing and a novel binning strategy. Biotechnol Biofuels 2016, 9, 26; 10.1186/s13068-016-0441-1 [doi].

6. Wommack, K.E.; Bhavsar, J.; Ravel, J. Metagenomics: read length matters. Appl. Environ. Microbiol. 2008, 74 (5), 1453-1463; 10.1128/AEM.02181-07 [doi].

7. Thomas, T.; Gilbert, J.; Meyer, F. Metagenomics-a guide from sampling to data analysis. Microb Inform $\operatorname{Exp}$ 2012, 2 (3), 1-12.

8. Ben-Amor, K.; Heilig, H.; Smidt, H.; Vaughan, E.E.; Abee, T.; de Vos, W.M. Genetic diversity of viable, injured, and dead fecal bacteria assessed by fluorescence-activated cell sorting and 16S rRNA gene analysis. Appl. Environ. Microbiol. 2005, 71 (8), 4679-4689; 71/8/4679 [pii].

9. Zakrzewski, M.; Goesmann, A.; Jaenicke, S.; Junemann, S.; Eikmeyer, F.; Szczepanowski, R.; Al-Soud, W.A.; Sorensen, S.; Puhler, A.; Schluter, A. Profiling of the metabolically active community from a production-scale biogas plant by means of high-throughput metatranscriptome sequencing. J. Biotechnol. 2012, 158 (4), 248-258; 10.1016/j.jbiotec.2012.01.020 [doi].

10. Bremges, A.; Maus, I.; Belmann, P.; Eikmeyer, F.; Winkler, A.; Albersmeier, A.; Puhler, A.; Schluter, A.; Sczyrba, A. Deeply sequenced metagenome and metatranscriptome of a biogas-producing microbial community from an agricultural production-scale biogas plant. Gigascience 2015, 4, 33-015-0073-6. eCollection 2015; 10.1186/s13742-015-0073-6 [doi].

11. Stewart, F.J.; Ottesen, E.A.; DeLong, E.F. Development and quantitative analyses of a universal rRNAsubtraction protocol for microbial metatranscriptomics. The ISME journal 2010, 4 (7), 896-907. 
12. Schink, B. Energetics of syntrophic cooperation in methanogenic degradation. Microbiol. Mol. Biol. Rev. 1997, 61 (2), 262-280.

13. Sousa, D.Z.; Pereira, M.A.; Smidt, H.; Stams, A.J.; Alves, M.M. Molecular assessment of complex microbial communities degrading long chain fatty acids in methanogenic bioreactors. FEMS Microbiol. Ecol. 2007, 60 (2), 252-265; FEM291 [pii].

14. McInerney, M. The genus Syntrophomonas, and other syntrophic bacteria. The prokaryotes 1992, , 20482057.

15. Zhao, H.; Yang, D.; Woese, C.R.; Bryant, M.P. Assignment of fatty acid-beta-oxidizing syntrophic bacteria to Syntrophomonadaceae fam. nov. on the basis of 16S rRNA sequence analyses. Int. J. Syst. Bacteriol. 1993, 43 (2), 278-286; 10.1099/00207713-43-2-278 [doi].

16. Wu, C.; Liu, X.; Dong, X. Syntrophomonas erecta subsp. sporosyntropha subsp. nov., a spore-forming bacterium that degrades short chain fatty acids in co-culture with methanogens. Syst. Appl. Microbiol. 2006, 29 (6), 457-462.

17. Jackson, B.E.; Bhupathiraju, V.K.; Tanner, R.S.; Woese, C.R.; McInerney, M.J. Syntrophus aciditrophicus sp. nov., a new anaerobic bacterium that degrades fatty acids and benzoate in syntrophic association with hydrogen-using microorganisms. Arch. Microbiol. 1999, 171 (2), 107-114.

18. Koops, H.; Purkhold, U.; Pommerening-Röser, A.; Timmermann, G.; Wagner, M. The prokaryotes: an evolving electronic resource for the microbiological community. 2003, .

19. Elberson, M.A.; Sowers, K.R. Isolation of an aceticlastic strain of Methanosarcina siciliae from marine canyon sediments and emendation of the species description for Methanosarcina siciliae. Int. J. Syst. Bacteriol. 1997, 47 (4), 1258-1261; 10.1099/00207713-47-4-1258 [doi].

20. Ma, K.; Liu, X.; Dong, X. Methanosaeta harundinacea sp. nov., a novel acetate-scavenging methanogen isolated from a UASB reactor. Int. J. Syst. Evol. Microbiol. 2006, 56 (Pt 1), 127-131; 56/1/127 [pii]. 
21. Kato, S.; Kosaka, T.; Watanabe, K. Substrate-dependent transcriptomic shifts in Pelotomaculum thermopropionicum grown in syntrophic co-culture with Methanothermobacter thermautotrophicus. Microb. Biotechnol. 2009, 2 (5), 575-584; 10.1111/j.1751-7915.2009.00102.x [doi].

22. Plugge, C.M.; Scholten, J.C.; Culley, D.E.; Nie, L.; Brockman, F.J.; Zhang, W. Global transcriptomics analysis of the Desulfovibrio vulgaris change from syntrophic growth with Methanosarcina barkeri to sulfidogenic metabolism. Microbiology 2010, 156 (Pt 9), 2746-2756; 10.1099/mic.0.038539-0 [doi].

23. Walker, C.B.; He, Z.; Yang, Z.K.; Ringbauer, J.A.,Jr; He, Q.; Zhou, J.; Voordouw, G.; Wall, J.D.; Arkin, A.P.; Hazen, T.C.; Stolyar, S.; Stahl, D.A. The electron transfer system of syntrophically grown Desulfovibrio vulgaris. J. Bacteriol. 2009, 191 (18), 5793-5801; 10.1128/JB.00356-09 [doi].

24. Nielsen, H.B.; Ahring, B.K. Responses of the biogas process to pulses of oleate in reactors treating mixtures of cattle and pig manure. Biotechnol. Bioeng. 2006, 95 (1), 96-105; 10.1002/bit.20963 [doi].

25. Palatsi, J.; Laureni, M.; Andrés, M.; Flotats, X.; Nielsen, H.; Angelidaki, I. Recovery strategies from long-chain fatty acids inhibition in anaerobic thermophilic digestion of manure. Bioresour. Technol. 2009, $100(20), 4588-4596$.

26. Cavaleiro, A.J.; Salvador, A.F.; Alves, J.I.; Alves, M. Continuous high rate anaerobic treatment of oleic acid based wastewater is possible after a step feeding start-up. Environ. Sci. Technol. 2009, 43 (8), 29312936.

27. Bassani, I.; Kougias, P.G.; Treu, L.; Angelidaki, I. Biogas Upgrading via Hydrogenotrophic Methanogenesis in Two-Stage Continuous Stirred Tank Reactors at Mesophilic and Thermophilic Conditions. Environ. Sci. Technol. 2015, 49 (20), 12585-12593; 10.1021/acs.est.5b03451 [doi].

28. Bolger, A.M.; Lohse, M.; Usadel, B. Trimmomatic: a flexible trimmer for Illumina sequence data. Bioinformatics 2014, 30 (15), 2114-2120; 10.1093/bioinformatics/btu170 [doi]. 
29. Glass, E.M.; Meyer, F. The metagenomics RAST server: a public resource for the automatic phylogenetic and functional analysis of metagenomes. Handbook of Molecular Microbial Ecology I: Metagenomics and Complementary Approaches 2011, ,325-331.

30. Langmead, B.; Salzberg, S.L. Fast gapped-read alignment with Bowtie 2. Nat. Methods 2012, 9 (4), 357 $359 ; 10.1038 /$ nmeth. 1923 [doi].

31. Anders, S.; Pyl, P.T.; Huber, W. HTSeq--a Python framework to work with high-throughput sequencing data. Bioinformatics 2015, 31 (2), 166-169; 10.1093/bioinformatics/btu638 [doi].

32. Zhou, X.; Lindsay, H.; Robinson, M.D. Robustly detecting differential expression in RNA sequencing data using observation weights. Nucleic Acids Res. 2014, 42 (11), e91; 10.1093/nar/gku310 [doi].

33. Quinlan, A.R.; Hall, I.M. BEDTools: a flexible suite of utilities for comparing genomic features. Bioinformatics 2010, 26 (6), 841-842; 10.1093/bioinformatics/btq033 [doi].

34. Kanehisa, M.; Goto, S.; Sato, Y.; Furumichi, M.; Tanabe, M. KEGG for integration and interpretation of large-scale molecular data sets. Nucleic Acids Res. 2012, 40 (Database issue), D109-14; 10.1093/nar/gkr988 [doi].

35. Treu, L.; Campanaro, S.; Nadai, C.; Toniolo, C.; Nardi, T.; Giacomini, A.; Valle, G.; Blondin, B.; Corich, V. Oxidative stress response and nitrogen utilization are strongly variable in Saccharomyces cerevisiae wine strains with different fermentation performances. Appl. Microbiol. Biotechnol. 2014, 98 (9), 4119-4135; 10.1007/s00253-014-5679-6 [doi].

36. Eikmeyer, F.G.; Rademacher, A.; Hanreich, A.; Hennig, M.; Jaenicke, S.; Maus, I.; Wibberg, D.; Zakrzewski, M.; Pühler, A.; Klocke, M. Detailed analysis of metagenome datasets obtained from biogasproducing microbial communities residing in biogas reactors does not indicate the presence of putative pathogenic microorganisms. Biotechnol Biofuels 2013, 6 (1), 49. 
37. Sousa, D.Z.; Pereira, M.A.; Stams, A.J.; Alves, M.M.; Smidt, H. Microbial communities involved in anaerobic degradation of unsaturated or saturated long-chain fatty acids. Appl. Environ. Microbiol. 2007, 73 (4), 1054-1064; AEM.01723-06 [pii].

38. Leimena, M.M.; Ramiro-Garcia, J.; Davids, M.; van den Bogert, B.; Smidt, H.; Smid, E.J.; Boekhorst, J.; Zoetendal, E.G.; Schaap, P.J.; Kleerebezem, M. A comprehensive metatranscriptome analysis pipeline and its validation using human small intestine microbiota datasets. BMC Genomics 2013, 14, 530-2164-14-530; 10.1186/1471-2164-14-530 [doi].

39. Peris-Bondia, F.; Latorre, A.; Artacho, A.; Moya, A.; D'Auria, G. The active human gut microbiota differs from the total microbiota. PLoS One 2011, 6 (7), e22448; 10.1371/journal.pone.0022448 [doi].

40. Hatamoto, M.; Imachi, H.; Yashiro, Y.; Ohashi, A.; Harada, H. Diversity of anaerobic microorganisms involved in long-chain fatty acid degradation in methanogenic sludges as revealed by RNA-based stable isotope probing. Appl. Environ. Microbiol. 2007, 73 (13), 4119-4127; AEM.00362-07 [pii].

41. Sousa, D.Z.; Salvador, A.F.; Ramos, J.; Guedes, A.P.; Barbosa, S.; Stams, A.J.; Alves, M.M.; Pereira, M.A. Activity and viability of methanogens in anaerobic digestion of unsaturated and saturated long-chain fatty acids. Appl. Environ. Microbiol. 2013, 79 (14), 4239-4245; 10.1128/AEM.00035-13 [doi].

42. Hattori, S.; Galushko, A.S.; Kamagata, Y.; Schink, B. Operation of the CO dehydrogenase/acetyl coenzyme A pathway in both acetate oxidation and acetate formation by the syntrophically acetate-oxidizing bacterium Thermacetogenium phaeum. J. Bacteriol. 2005, 187 (10), 3471-3476; 187/10/3471 [pii].

43. Lee, M.J.; Zinder, S.H. Isolation and Characterization of a Thermophilic Bacterium Which Oxidizes Acetate in Syntrophic Association with a Methanogen and Which Grows Acetogenically on $\mathrm{H}(2)-\mathrm{CO}(2)$. Appl. Environ. Microbiol. 1988, 54 (1), 124-129.

44. Schnürer, A.; Svensson, B.H.; Schink, B. Enzyme activities in and energetics of acetate metabolism by the mesophilic syntrophically acetate-oxidizing anaerobe Clostridium ultunense. FEMS Microbiol. Lett. 1997, $154(2), 331-336$. 
45. Schnurer, A.; Nordberg, A. Ammonia, a selective agent for methane production by syntrophic acetate oxidation at mesophilic temperature. Water Sci. Technol. 2008, 57 (5), 735-740; 10.2166/wst.2008.097 [doi].

46. Westerholm, M.; Leven, L.; Schnurer, A. Bioaugmentation of syntrophic acetate-oxidizing culture in biogas reactors exposed to increasing levels of ammonia. Appl. Environ. Microbiol. 2012, 78 (21), 7619 7625; 10.1128/AEM.01637-12 [doi].

47. Hattori, S.; Kamagata, Y.; Hanada, S.; Shoun, H. Thermacetogenium phaeum gen. nov., sp. nov., a strictly anaerobic, thermophilic, syntrophic acetate-oxidizing bacterium. Int. J. Syst. Evol. Microbiol. 2000, 50 Pt 4, 1601-1609; 10.1099/00207713-50-4-1601 [doi].

48. Kawai, M.; Yamada, S.; Ishidoshiro, A.; Oyamada, Y.; Ito, H.; Yamagishi, J. Cell-wall thickness: possible mechanism of acriflavine resistance in meticillin-resistant Staphylococcus aureus. J. Med. Microbiol. 2009, 58 (Pt 3), 331-336; 10.1099/jmm.0.004184-0 [doi].

49. Sasatsu, M.; Shima, K.; Shibata, Y.; Kono, M. Nucleotide sequence of a gene that encodes resistance to ethidium bromide from a transferable plasmid in Staphylococcus aureus. Nucleic Acids Res. 1989, 17 (23), 10103.

50. Cui, L.; Murakami, H.; Kuwahara-Arai, K.; Hanaki, H.; Hiramatsu, K. Contribution of a thickened cell wall and its glutamine nonamidated component to the vancomycin resistance expressed by Staphylococcus aureus Mu50. Antimicrob. Agents Chemother. 2000, 44 (9), 2276-2285.

51. Sieradzki, K.; Tomasz, A. Alterations of cell wall structure and metabolism accompany reduced susceptibility to vancomycin in an isogenic series of clinical isolates of Staphylococcus aureus. J. Bacteriol. 2003, 185 (24), 7103-7110.

52. Sheu, C.W.; Freese, E. Lipopolysaccharide layer protection of gram-negative bacteria against inhibition by long-chain fatty acids. J. Bacteriol. 1973, 115 (3), 869-875. 
53. Miller, R.M.; Tomaras, A.P.; Barker, A.P.; Voelker, D.R.; Chan, E.D.; Vasil, A.I.; Vasil, M.L. Pseudomonas aeruginosa twitching motility-mediated chemotaxis towards phospholipids and fatty acids: specificity and metabolic requirements. J. Bacteriol. 2008, 190 (11), 4038-4049; 10.1128/JB.00129-08 [doi].

54. Wang, W.; Shao, Z. The long-chain alkane metabolism network of Alcanivorax dieselolei. Nat. Commun. 2014, 5, 5755; 10.1038/ncomms6755 [doi].

55. Blackhart, B.D.; Zusman, D.R. "Frizzy" genes of Myxococcus xanthus are involved in control of frequency of reversal of gliding motility. Proc. Natl. Acad. Sci. U. S. A. 1985, 82 (24), 8767-8770.

56. Martin, A.C.; Wadhams, G.H.; Armitage, J.P. The roles of the multiple CheW and CheA homologues in chemotaxis and in chemoreceptor localization in Rhodobacter sphaeroides. Mol. Microbiol. 2001, 40 (6), 1261-1272; mmi2468 [pii].

57. Szurmant, H.; Ordal, G.W. Diversity in chemotaxis mechanisms among the bacteria and archaea. Microbiol. Mol. Biol. Rev. 2004, 68 (2), 301-319; 10.1128/MMBR.68.2.301-319.2004 [doi].

58. Baker, M.D.; Wolanin, P.M.; Stock, J.B. Signal transduction in bacterial chemotaxis. Bioessays 2006, 28 (1), 9-22.

59. Kirby, J.R. Chemotaxis-like regulatory systems: unique roles in diverse bacteria. Annu. Rev. Microbiol. 2009, 63, 45-59; 10.1146/annurev.micro.091208.073221 [doi].

60. Liu, J.; Hu, B.; Morado, D.R.; Jani, S.; Manson, M.D.; Margolin, W. Molecular architecture of chemoreceptor arrays revealed by cryoelectron tomography of Escherichia coli minicells. Proc. Natl. Acad. Sci. U. S. A. 2012, 109 (23), E1481-8; 10.1073/pnas.1200781109 [doi].

61. Hazelbauer, G.L.; Falke, J.J.; Parkinson, J.S. Bacterial chemoreceptors: high-performance signaling in networked arrays. Trends Biochem. Sci. 2008, 33 (1), 9-19; 10.1016/j.tibs.2007.09.014 [doi]. 